\title{
Intersections of Translation of a Class of Self-Affine Sets
}

\author{
Jian Lu, Yuru Zou, and Lijing Wang \\ College of Mathematics and Computational Science, Shenzhen University, Shenzhen 518060, China \\ Correspondence should be addressed to Yuru Zou; yrzou@163.com
}

Received 20 September 2013; Revised 17 December 2013; Accepted 17 December 2013

Academic Editor: Naseer Shahzad

Copyright (c) 2013 Jian Lu et al. This is an open access article distributed under the Creative Commons Attribution License, which permits unrestricted use, distribution, and reproduction in any medium, provided the original work is properly cited.

Intersections of translation of a class of self-affine sets are investigated by constructing sofic affine-invariant sets which coincide with these sets and then a scheme for computing the Hausdorff dimensions of these intersections is given.

\section{Introduction}

Fix integers $2 \leq m \leq n$. Let $T$ be the expanding endomorphism of the 2-torus $\mathbb{T}^{2}=\mathbb{R}^{2} / \mathbb{Z}^{2}$ given by the matrix $\operatorname{diag}(n, m)$ and let $D \subseteq I \times J$ be the set of digits with $I=$ $\{0,1, \ldots, n-1\}, J=\{0,1, \ldots, m-1\}$. The set $K_{T, D}$ is defined as follows:

$$
K_{T, D}=\left\{\sum_{k=1}^{\infty} T^{-k} d_{k}: d_{k} \in D \forall k \geq 1\right\},
$$

which can be viewed as the unique invariant set of a family of $N(=\# D)$ affine contractions as follows:

$$
F_{j}\left(\begin{array}{l}
x \\
y
\end{array}\right)=T^{-1}\left(\left(\begin{array}{l}
x \\
y
\end{array}\right)+d_{j}\right), \quad d_{j} \in D, j=1,2, \ldots, N .
$$

If no confusion arises about $T, D$, we will write $K$ instead of $K_{T, D}$. Throughout this paper, we let \#D denote the cardinality of $D$. The set $K$, called McMullen's self-affine carpet, was first studied by McMullen [1] and Bedford [2], independently, to determine its Hausdorff and box-counting dimensions. From then on, there has been a fast growth in general interest in the study of McMullen's self-affine carpet $K$. Its packing and Hausdorff measures were explored in $[3,4]$. The results of $[1,2]$ were extended to the compact subsets of the 2-torus corresponding to shifts of finite type or sofic shifts and to the Sierpinski sponges by Kenyon and Peres [5, 6]. Gui and Li [7] studied a class of subset of Sierpinski carpets for which the allowed digits in the expansions fall into each fiber set with a prescribed frequency. Hua [8] investigated a class of selfaffine sets with overlaps and showed that they can be viewed as sofic affine invariant sets without overlaps.
Intersection of Cantor sets has been the subject of several studies [9-19], the context and motivation being numerous. Davis and $\mathrm{Hu}$ [12] explored the Hausdorff dimension of the intersection of two middle third Cantor sets and showed that the Hausdorff dimension can take any value from 0 to $\ln 2 / \ln 3$. Williams [10] produced examples to show that the intersection of two Cantor sets can vary from being one point to containing another Cantor set. Li and Xiao [13] showed that intersection resulting from two middle- $\alpha$ sets being translated across one another is a generalized Moran set if the contraction lies in $[0,1 / 3]$, whose Hausdorff, packing, upper Box dimension then can be obtained by the results of the Moran set. Nekka and $\mathrm{Li}[14,15]$ studied the properties of intersection of Cantor sets with their translations. Dai and Tian $[16,17]$ got the related properties of a class of self-similar sets in the plane.

In this paper, we develop a new algorithm to explore the structure of $K \cap(K+t)$ for $t \in K-K$, which extends our previous results in [18] and is different from the listed papers. It is easy to see that $K \cap(K+t) \neq \emptyset$ if and only if $t \in K-K$; so throughout this paper, we always suppose that $t \in K-K$. The intersection, generally, has a complicated structure which leads the set quite different from McMullen's self-affine carpet. The main difficulty in the study of the structure of the intersection comes from the fact that the number of affine contractions used in the construction may vary from step to step. However, by defining a suitable equivalence relation on the basic rectangles of $K \cap(K+t)$ and partitioning them into equivalence classes, a sofic affine-invariant set coinciding with $K \cap(K+t)$ then can be constructed, if the number of equivalence classes is finite. Our idea of the construction of the sofic affine-invariant set is enlightened by [8, 20-22]. 
To state our main result, we need to recall some definitions and results about the sofic affine-invariant set. For details, one can refer to $[6,9]$.

Let $G=(\mathscr{V}, \mathscr{E})$ be a finite directed graph in which loops and multiple edges are allowed. Let $D_{0}=\{0,1, \ldots, n-1\} \times$ $\{0,1, \ldots, m-1\}$ be the set of symbols. Suppose that the edges of $G$ are labeled in symbols in $D_{0}$ in a right resolving fashion: no two edges emanating from the same vertex have the same symbol. Then the symbol sequences which arise from infinite paths in $G$ form a sofic system on $\# D_{0}$ symbols. That is,

$$
\Theta=\left\{\left\{d_{k}\right\}_{k \geq 1}:\left\{d_{k}\right\}_{k \geq 1}\right.
$$

is an infinite path in $G$, where $\left.d_{k} \in D_{0}\right\}$.

Suppose that $\Theta \subset D_{0}^{\mathbb{N}}$ is the resulting sofic system. We call

$$
\Gamma_{T}(\Theta)=\left\{\sum_{k=1}^{\infty} T^{-k} d_{k}:\left\{d_{k}\right\}_{k \geq 1} \in \Theta\right\}
$$

a $T$-invariant sofic set. An adjacency matrix $A$, according to $G=(\mathscr{V}, \mathscr{E})$, is constructed, where, for two vertices $v_{1}, v_{2}$ in $\mathscr{V}, A\left(v_{1}, v_{2}\right)$ is the number of edges in $\mathscr{E}$ from $v_{1}$ to $v_{2}$.

Theorem 1. Suppose that $t=\left(p_{1} / q_{1}, p_{2} / q_{2}\right) \in \mathbb{Q}^{2}$. Then

$$
K \cap(K+t)=\Gamma_{T}(\Theta) .
$$

To compute the Hausdorff dimension of $K \cap(K+t)$, the following result in [6] is needed.

Kenyon-Peres's Theorem (see [6, Theorem 3.2]). Let $G=$ $(\mathscr{V}, \mathscr{E})$ be a finite directed graph with edges labeled by $D_{0}$ and adjacency matrix $A$. Let $\Theta \subset D_{0}^{\mathbb{N}}$ be the resulting sofic system. Then the Hausdorff dimension of $\Gamma_{T}(\Theta)$ is given by

$$
\begin{aligned}
\operatorname{dim}_{H} & \left(\Gamma_{T}(\Theta)\right) \\
& =\lim _{k \rightarrow \infty} \log _{m} \sum_{0 \leq i_{1}, \ldots, i_{k} \leq m-1}\left\|A_{i_{k}} \cdot A_{i_{k-1}} \cdot \ldots \cdot A_{i_{1}}\right\|^{\alpha},
\end{aligned}
$$

where, for $0 \leq j<m$ and vertices $v_{1}, v_{2}$ in $G, A_{j}\left(v_{1}, v_{2}\right)$ denotes the number of edges from $v_{1}$ to $v_{2}$ in $G$ such that the second coordinate of their label is $j, \alpha=\log m / \log n$.

This paper is arranged as follows. A description for equivalence classes on $K \cap(K+t)$ is arranged in Section 2. A sofic affine-invariant set coinciding with $K \cap(K+t)$ is then constructed in Section 3, if the number of equivalence classes is finite (see Theorem 6). A sufficient condition for the number of equivalence classes to be finite is also established in Section 3 (see Proposition 7). Finally, the proof of the Theorem 1 is completed in this section. Some examples are included in Section 4.

\section{Equivalence Classes on $K \cap(K+t)$}

In this section we consider the geometrical structure of $K \cap$ $(K+t)(\neq \emptyset)$. First we define a suitable equivalence relation on the basic rectangles of $K \cap(K+t)$ and partition them into equivalence classes.
Let $\left\{F_{j}\right\}_{j=1}^{N}$ be defined as in (2); simple computation yields

$$
K+t=\bigcup_{j=1}^{N}\left(T^{-1}(K+t)+T^{-1} d_{j}+t-T^{-1} t\right) .
$$

Then define

$$
S_{j}\left(\begin{array}{l}
x \\
y
\end{array}\right)=T^{-1}\left(\left(\begin{array}{l}
x \\
y
\end{array}\right)+d_{j}+T t-t\right), \quad j=1, \ldots, N,
$$

such that $K+t$ can be viewed as the unique invariant nonempty compact set under mappings $S_{j}, j=1, \ldots, N$. Let $O=$ $t+(0,1)^{2},\left\{S_{j}\right\}_{j=1}^{N}$, obviously, satisfy the OSC. Set

$$
D^{*}=\left\{d_{j}+T t-t, d_{j} \in D\right\} .
$$

In order to analyze the structure of $K \cap(K+t)$, we get the following standard notations.

Let $\Sigma=\{1, \ldots, N\}$ and $\Sigma^{*}=\cup_{p \geq 0} \Sigma^{p}$ be the set of all finite words, where $\Sigma^{p}$ is the set of all words of length $p$, with $\Sigma^{0}$ containing only the empty word $\emptyset . \Sigma^{\mathbb{N}}=\left\{i_{1} i_{2} \cdots: i_{n} \in\right.$ $\Sigma$ for all $1 \leq n<\infty$. For $J \in \Sigma^{p}$, let $|J|=p$ denote the length of $J$. For $I \in \Sigma^{p}$ and $J \in \Sigma^{q}$, let $I J$ be the concatenation of $I$ and $J$. For $I=i_{1} i_{2} \cdots \in \Sigma^{\mathbb{N}}$ and a positive integer $k$, let $I \mid k=i_{1} \cdots i_{k}$ denote the truncation of $I$ to the $k$ th place.

We define the iterated mappings using these notations. Let $J=\left(j_{1}, \ldots, j_{k}\right) \in \Sigma^{k}$. Then

$$
\begin{gathered}
F_{J}=F_{j_{1}} \circ F_{j_{2}} \circ \cdots \circ F_{j_{k}}, \\
S_{J}=S_{j_{1}} \circ S_{j_{2}} \circ \cdots \circ S_{j_{k}}
\end{gathered}
$$

with $F_{\emptyset}=S_{\emptyset}=I$, the identity map on $\mathbb{R}^{2}$. Thus we get

$$
\begin{gathered}
K=\bigcap_{k=1}^{\infty} \bigcup_{J \in \Sigma^{k}} F_{J}(\Omega), \\
K+t=\bigcap_{k=1}^{\infty} \bigcup_{J \in \Sigma^{k}} S_{J}\left(\Omega^{t}\right),
\end{gathered}
$$

where $\Omega=[0,1]^{2}$ and $\Omega^{t}=t+[0,1]^{2}$. We call $F_{J}(\Omega), S_{J}\left(\Omega^{t}\right)$ the basic rectangles of rank $k$, respectively. For simplicity without confusion, we further call them basick-rectangles and we write $F_{J}(\Omega)=\Omega_{J}$ and $S_{J}\left(\Omega^{t}\right)=\Omega_{J}^{t}$ in this paper.

Suppose that there exist $I \in \Sigma^{k}, J \in \Sigma^{k+1}$, and $\ell \in \Sigma^{1}$ such that

$$
\tau_{1}=\Omega_{I}, \quad \tau_{2}=\Omega_{J}, \quad J=I \ell .
$$

Then we connect a directed edge $\ell$ from $\tau_{1}$ to $\tau_{2}$ and denote it by $\tau_{1} \stackrel{\ell}{\rightarrow} \tau_{2}$. We call $\tau_{1}$ the parent of $\tau_{2}$ and $\tau_{2}$ an offspring of $\tau_{1}$. Definitions of parent and offspring can be parallel extended to $K+t$.

Following we investigate the relationships between the basic $k$-rectangles of $K$ and $K+t$.

Suppose that $\Omega_{J}, J \in \Sigma^{k}, k \geq 0$; the neighborhood of $\Omega_{J}$ with respect to the basic $k$-rectangles of $K+t$ is defined as

$$
N\left(\Omega_{J}\right)=\left\{\Omega_{I}^{t}: I \in \Sigma^{k}, \Omega_{J} \cap \Omega_{I}^{t} \neq \emptyset\right\} .
$$


Let $\Lambda_{k}=\left\{J \in \Sigma^{k}: N\left(\Omega_{J}\right) \neq \emptyset, k \geq 0\right\}$. Note that, for some $\Omega_{J}$, $J \in \Sigma^{k}, N\left(\Omega_{J}\right)$ may be empty; namely, these basic $k$-rectangles do not meet any basic $k$-rectangles of $K+t$, and then they have nothing to do with the intersection $K \cap(K+t)$, which will be deleted. So let

$$
\mathscr{M}_{k}=\left\{\Omega_{J}, J \in \Lambda_{k}\right\} .
$$

The basic $k$-rectangles of $K$ and $K+t$ are completely determined by their left-lower corner points. For this point, if $\tau \in \mathscr{M}_{k}$ we define

$$
\begin{gathered}
R_{k}(\tau)=\left\{T^{k}(\widehat{\delta}-\widehat{\tau}): \delta \in N(\tau)\right\}, \\
R=\left\{R_{k}(\tau): \tau \in \mathscr{M}_{k}, k \geq 0\right\},
\end{gathered}
$$

where $\widehat{\delta}, \widehat{\tau}$ are the left-lower corner points of $\delta$, $\tau$, respectively. For $\tau_{1} \in \mathscr{M}_{k_{1}}$ and $\tau_{2} \in \mathscr{M}_{k_{2}}$, we say that $\tau_{1}$ and $\tau_{2}$ are equivalent, if $R_{k_{1}}\left(\tau_{1}\right)=R_{k_{2}}\left(\tau_{2}\right)$. Next we prove a recurrent relationship on $R$.

Let $\tau_{1}=\Omega_{I}$ and $\tau_{2}=\Omega_{J}$, where $I \in \Sigma^{k_{1}}, J \in \Sigma^{k_{2}}$. Suppose that $R_{k_{1}}\left(\tau_{1}\right)=R_{k_{2}}\left(\tau_{2}\right)$ and set

$$
\begin{aligned}
& N\left(\tau_{1}\right)=\left\{v_{1}, \ldots, v_{t}\right\}, \\
& N\left(\tau_{2}\right)=\left\{u_{1}, \ldots, u_{t}\right\},
\end{aligned}
$$

such that $T^{k_{1}}\left(\widehat{v}_{i}-\widehat{\tau}_{1}\right)=T^{k_{2}}\left(\widehat{u}_{i}-\widehat{\tau}_{2}\right), 1 \leq i \leq t$. Let $\sigma_{\mathbf{j}}^{1}, \sigma_{\mathbf{j}}^{2}$ be the offsprings of $\tau_{1}$ and $\tau_{2}$ connected by edge $\mathbf{j} \in \Sigma^{1}$, respectively, and denote them by $\tau_{1} \stackrel{\mathfrak{j}}{\rightarrow} \sigma_{\mathbf{j}}^{1}, \tau_{2} \stackrel{\mathbf{j}}{\rightarrow} \sigma_{\mathbf{j}}^{2}$. Similarly, $v_{\mathbf{k}}^{i}, u_{\mathbf{k}}^{i}$ are the offsprings of $v_{i}$ and $u_{i}$ connected by edge $\mathbf{k} \in \Sigma^{1}$, respectively. That is $v_{i} \stackrel{\mathbf{k}}{\rightarrow} v_{\mathbf{k}}^{i}, u_{i} \stackrel{\mathbf{k}}{\rightarrow} u_{\mathbf{k}}^{i}$.

Lemma 2. With above notations, let $g: \mathbb{R}^{2} \rightarrow \mathbb{R}^{2}$ be an affine mapping,

$$
g(x)=T^{k_{1}-k_{2}} x+\widehat{\tau}_{2}-T^{k_{1}-k_{2}} \widehat{\tau}_{1},
$$

where $\widehat{\tau}_{1}, \widehat{\tau}_{2}$ are the left-lower corner points of $\tau_{1}$ and $\tau_{2}$, respectively. Then $\sigma_{\mathbf{j}}^{2}=g\left(\sigma_{\mathbf{j}}^{1}\right)$. If $R_{k_{1}}\left(\tau_{1}\right)=R_{k_{2}}\left(\tau_{2}\right)$, then $u_{i}=g\left(v_{i}\right), i=1, \ldots, t$. Furthermore $u_{\mathbf{k}}^{i}=g\left(v_{\mathbf{k}}^{i}\right)$.

Proof. Note that

$$
\begin{aligned}
& \sigma_{\mathbf{j}}^{1}=T^{-k_{1}-1}(\Omega)+\widehat{\tau}_{1}+d_{\mathbf{j}} T^{-k_{1}-1}, \\
& \sigma_{\mathbf{j}}^{2}=T^{-k_{2}-1}(\Omega)+\widehat{\tau}_{2}+d_{\mathbf{j}} T^{-k_{2}-1},
\end{aligned}
$$

where $d_{\mathbf{j}} \in D$. Then

$$
\begin{aligned}
g\left(\sigma_{\mathbf{j}}^{1}\right)= & T^{-k_{2}-1}(\Omega)+T^{k_{1}-k_{2}} \widehat{\tau}_{1}+d_{\mathbf{j}} T^{-k_{2}-1} \\
& +\widehat{\tau}_{2}-T^{k_{1}-k_{2}} \widehat{\tau}_{1}=\sigma_{\mathbf{j}}^{2} .
\end{aligned}
$$

We now prove that $u_{i}=g\left(v_{i}\right), i=1, \ldots, t . \widehat{\tau}_{2}-T^{k_{1}-k_{2}} \widehat{\tau}_{1}=$ $\widehat{u}_{i}-T^{k_{1}-k_{2}} \widehat{v}_{i}$ is the direct conclusion from $R_{k_{1}}\left(\tau_{1}\right)=R_{k_{2}}\left(\tau_{2}\right)$, which yields $u_{i}=g\left(v_{i}\right), i=1, \ldots, m \cdot u_{\mathbf{k}}^{i}=g\left(v_{\mathbf{k}}^{i}\right)$ can be easily obtained by the same argument as $\sigma_{\mathbf{j}}^{2}=g\left(\sigma_{\mathfrak{j}}^{1}\right)$.
Proposition 3. With above notations, if $R_{k_{1}}\left(\tau_{1}\right)=R_{k_{2}}\left(\tau_{2}\right)$, then, for $\mathbf{j} \in \Sigma^{1}, \sigma_{\mathbf{j}}^{1} \in \mathscr{M}_{k_{1}+1}$ if and only if $\sigma_{\mathbf{j}}^{2} \in \mathscr{M}_{k_{2}+1}$. If $\sigma_{\mathbf{j}}^{1} \in \mathscr{M}_{k_{1}+1}$, then $R_{k_{1}+1}\left(\sigma_{\mathbf{j}}^{1}\right)=R_{k_{2}+1}\left(\sigma_{\mathbf{j}}^{2}\right)$.

Proof. By Lemma 2, we have

$$
v_{\mathbf{k}}^{i} \cap \sigma_{\mathbf{j}}^{1} \neq \emptyset \Longleftrightarrow g\left(v_{\mathbf{k}}^{i}\right) \cap g\left(\sigma_{\mathbf{j}}^{1}\right)=u_{\mathbf{k}}^{i} \cap \sigma_{\mathbf{j}}^{2} \neq \emptyset,
$$

so $\sigma_{j}^{1} \in \mathscr{M}_{k_{1}+1} \Leftrightarrow \sigma_{j}^{2} \in \mathscr{M}_{k_{2}+1}$.

One can check that

$$
\begin{aligned}
& N\left(\sigma_{\mathbf{j}}^{1}\right)=\left\{v_{\mathbf{k}}^{i}: v_{i} \in N\left(\tau_{1}\right), v_{\mathbf{k}}^{i} \cap \sigma_{\mathbf{j}}^{1} \neq \emptyset\right\}, \\
& N\left(\sigma_{\mathbf{j}}^{2}\right)=\left\{u_{\mathbf{k}}^{i}: u_{i} \in N\left(\tau_{2}\right), u_{\mathbf{k}}^{i} \cap \sigma_{\mathbf{j}}^{2} \neq \emptyset\right\} .
\end{aligned}
$$

By Lemma 2 and (21), (22) can be rewritten as

$$
\begin{aligned}
N\left(\sigma_{\mathbf{j}}^{2}\right) & =\left\{g\left(v_{\mathbf{k}}^{i}\right): u_{i} \in N\left(\tau_{2}\right), u_{\mathbf{k}}^{i} \cap \sigma_{\mathbf{j}}^{2} \neq \emptyset\right\} \\
& =\left\{g\left(v_{\mathbf{k}}^{i}\right): v_{i} \in N\left(\tau_{1}\right), v_{\mathbf{k}}^{i} \cap \sigma_{\mathbf{j}}^{1} \neq \emptyset\right\} \\
& =\left\{g\left(v_{\mathbf{k}}^{i}\right): v_{\mathbf{k}}^{i} \in N\left(\sigma_{\mathbf{j}}^{1}\right)\right\} .
\end{aligned}
$$

Observe that

$$
\begin{gathered}
\widehat{\sigma}_{\mathbf{j}}^{1}=\widehat{\tau}_{1}+d_{\mathbf{j}} T^{-k_{1}-1}, \quad \widehat{\sigma}_{\mathbf{j}}^{2}=\widehat{\tau}_{2}+d_{\mathbf{j}} T^{-k_{2}-1}, \\
\widehat{v}_{\mathbf{k}}^{i}=\widehat{v}_{i}+c_{\mathbf{k}} T^{-k_{1}-1}+T^{-k_{1}} t\left(T^{-1}-1\right), \\
\widehat{u}_{\mathbf{k}}^{i}=\widehat{u}_{i}+c_{\mathbf{k}} T^{-k_{2}-1}+T^{-k_{2}} t\left(T^{-1}-1\right),
\end{gathered}
$$

where $c_{\mathrm{k}} \in D^{*}$ (defined as in (9)). By condition $R_{k_{1}}\left(\tau_{1}\right)=$ $R_{k_{2}}\left(\tau_{2}\right)$, we obtain

$$
T^{k_{2}+1}\left(\widehat{u}_{\mathbf{k}}^{i}-\widehat{\sigma}_{\mathbf{j}}^{2}\right)=T^{k_{1}+1}\left(\widehat{v}_{\mathbf{k}}^{i}-\widehat{\sigma}_{\mathbf{j}}^{1}\right) .
$$

Let $R$ be defined as in (16); if $R$ is a finite set, we then call that the types of the basic rectangles of $K \cap(K+t)$ is finite.

We remark that, for $t \in K-K$, if the types of the basic rectangles of $K \cap(K+t)$ is recursive, such as type $A$ produces types $B$, and $C, B$ produces $D, C$ produces $B$ and finally $D$ produces $A$, then by Proposition 3 , one can claim that the types of the basic rectangles of $K \cap(K+t)$ are finite.

From (11), and the definition of $\Lambda_{k}$, we have

$$
\begin{aligned}
K \cap(K+t) & =\left(\bigcap_{k=1}^{\infty} \bigcup_{J \in \Sigma^{k}} \Omega_{J}\right) \bigcap\left(\bigcap_{k=1}^{\infty} \bigcup_{I \in \Sigma^{k}} \Omega_{I}^{t}\right) \\
& =\bigcap_{k=1}^{\infty} \Pi_{k},
\end{aligned}
$$

where $\Pi_{k}=\left(\bigcup_{J \in \Lambda_{k}} \Omega_{J}\right) \bigcap\left(\bigcup_{I \in \Sigma^{k}} \Omega_{I}^{t}\right)$.

\section{Proposition 4.}

$$
K \cap(K+t)=\bigcap_{k=1 J \in \Lambda_{k}}^{\infty} \Omega_{J} .
$$


Proof. It is easy to see that $\Pi_{k} \subset \bigcup_{J \in \Lambda_{k}} \Omega_{J}$; therefore

$$
K \cap(K+t) \subseteq \bigcap_{k=1}^{\infty} \bigcup_{J \in \Lambda_{k}} \Omega_{J} .
$$

To prove the reverse inclusion, we let $\varepsilon / 2>0$; then for $k_{1}$, $k_{2}$ large enough, such that $\left|\Omega_{J}\right| \leq \varepsilon / 2$ for $J \in \Lambda_{k_{1}}$, and $\Pi_{k_{2}} \subseteq[K \cap(K+t)]_{\varepsilon / 2}(|A|$ denotes the diameter of $A$ and $[A]_{\varepsilon}$ the open $\varepsilon$-neighborhood of $A$ ). Let $k=\max \left\{k_{1}, k_{2}\right\}$, then $\max \left\{\left|\Omega_{J^{\prime}}\right|, J^{\prime} \in \Lambda_{k}\right\} \leq \varepsilon / 2\left(J^{\prime}\right.$ always exists; otherwise $K \cap(K+t)=\emptyset)$. On the other hand $\Omega_{J^{\prime}} \cap \Pi_{k} \neq \emptyset$, then $\Omega_{J^{\prime}} \subseteq[K \cap(K+t)]_{\varepsilon}$. Hence

$$
\bigcap_{k=1}^{\infty} \bigcup_{J^{\prime} \in \Lambda_{k}} \Omega_{J^{\prime}} \subseteq[K \bigcap(K+t)]_{\varepsilon} .
$$

The desired result is then obtained by letting $\varepsilon$ tend to 0 .

For some $J \in \Lambda_{k}$, it is possible that, for all $i \in \Sigma^{1}, J i \notin$ $\Lambda_{k+1}$; namely, all offsprings of $\Omega_{J}$ do not belong to $\mathscr{M}_{k+1}$. So set

$$
\begin{aligned}
& \Lambda=\left\{J=\left(j_{1}, j_{2}, \ldots, j_{k}, \ldots\right):\right. \\
& \left.\quad \text { for any } k \geq 0, J \mid k=\left(j_{1}, j_{2}, \ldots, j_{k}\right) \in \Lambda_{k}\right\} .
\end{aligned}
$$

Remark 5. We remark that, by Proposition $4, K \cap(K+t)$ can also be expressed in radix expansions as follows:

$$
K \cap(K+t)=\lim _{k \rightarrow \infty} \sum_{i=1}^{k} T^{-i} d_{l_{i}},
$$

where $d_{l_{i}} \in D, I=\left(l_{1}, \ldots, l_{k}, \ldots\right) \in \Lambda$.

\section{Sofic Affine-Invariant Set}

Suppose that $R$ is a finite set; let $\mathscr{V}=R=\left\{R_{k}(\tau): \tau \in\right.$ $\left.\mathscr{M}_{k} k \geq 0\right\}$. Set $\mathscr{V}=\left\{\omega, \omega_{1}, \ldots, \omega_{\mathrm{Q}}\right\}$ and $R_{0}(\Omega)=\omega$. We now define the directed edges $\mathscr{E}=\left\{E_{i, j}\right\}_{i, j=1}^{Q}$ on $\mathscr{V}$. Fix $i(1 \leq$ $i \leq Q)$ and a vertex $\tau=\Omega_{I} \in \mathscr{M}_{k}$ such that $R_{k}(\tau)=\omega_{i}$. Let $\delta_{\ell}=\Omega_{I \ell}$ in $\mathscr{M}_{k+1}$ be an offspring of $\tau$ connected by edge $\ell \in \Sigma^{1}$. Consider

$$
E_{i, j}=\left\{d_{\ell}: \tau \stackrel{\ell}{\longrightarrow} \delta_{\ell}, R_{k+1}\left(\delta_{\ell}\right)=\omega_{j}, d_{\ell} \in D\right\} .
$$

Note that if none of the offsprings of $\tau$ belongs to $\mathscr{M}_{k+1}$, then $E_{i, j}=\emptyset$, for all $j$. We discard those $\omega_{i}$ 's that there is no path going out and discard all the paths going to such discarded vertices. Hence, without loss of generality, we assume, that for each $i$,

$$
\sum_{j=1}^{Q} \# E_{i, j} \geq 1
$$

A finite directed graph $G=(\mathscr{V}, \mathscr{E})$ then is obtained. Let

$$
\Theta=\left\{\left\{d_{k}\right\}_{k \geq 1}:\left\{d_{k}\right\}_{k \geq 1}\right.
$$

is an infinite path in $G$, where $\left.d_{k} \in D\right\}$.
Then $\Theta \subset D^{\ell}$ is the resulting sofic system and

$$
\Gamma_{T}(\Theta)=\left\{\sum_{k=1}^{\infty} T^{-k} d_{k}:\left\{d_{k}\right\}_{k \geq 1} \in \Theta\right\}
$$

is a sofic invariant set.

Theorem 6. If $R$ is a finite set, then

$$
K \cap(K+t)=\Gamma_{T}(\Theta) .
$$

Proof. By Remark 5, $K \cap(K+t)$ can be expressed as

$$
K \cap(K+t)=\lim _{k \rightarrow \infty} \sum_{i=1}^{k} d_{l_{i}} T^{-i},
$$

where $d_{l_{i}} \in D, I=\left(l_{1}, \ldots, l_{k}, \ldots\right) \in \Lambda$. By the definition of $\Lambda$ in (30) and the construction of $G=(\mathscr{V}, \mathscr{E})$, one can claim that $d_{l_{1}} \cdots d_{l_{k}} \ldots$ is an infinite path in $G$ and then belongs to $\Theta$. Thus $K \cap(K+t) \subseteq \Gamma_{T}(\Theta)$. The inverse inclusion is left for the readers.

Following we give some sufficient condition for the set $R$ (defined as in (16)) to be finite.

Proposition 7. Suppose that $t=\left(p_{1} / q_{1}, p_{2} / q_{2}\right) \in \mathbb{Q}^{2}$. Then the set $R$ is finite.

Proof. Suppose that $\tau \in M_{k}, \delta \in N(\tau) . \widehat{\tau}, \widehat{\delta}$ denote the leftlower corner points of $\tau, \delta$ respectively; then

$$
\widehat{\tau}=\sum_{i=1}^{k} d_{\ell_{i}} T^{-i}
$$

where $d_{\ell_{i}} \in D$;

$$
\widehat{\delta}=\sum_{i=1}^{k} c_{\ell_{i}} T^{-i}+T^{-k} t,
$$

where $c_{\ell_{i}} \in D^{*}$. Consider

$$
\begin{aligned}
T^{k}(\widehat{\delta}-\widehat{\tau}) & =T^{k}\left(\sum_{i=1}^{k}\left(c_{\ell_{i}}-d_{\ell_{i}}\right) T^{-i}+T^{-k} t\right) \\
& =\frac{1}{q}\left(\sum_{i=1}^{k}\left(e_{\ell_{i}}+p T-p\right) T^{k-i}+p\right),
\end{aligned}
$$

where $p=\left(q p_{1} / q_{1}, q p_{2} / q_{2}\right) ; q$ is the least common multiple of $q_{1}$ and $q_{2} \cdot e_{\ell_{i}} \in q(D-D), q D:=\left\{q d_{j}, d_{j} \in D\right\}$. So the coordinates of $q T^{k}(\widehat{\delta}-\widehat{\tau})$ are integers. On the other hand $\tau \cap$ $\delta \neq \emptyset$ implies that

$$
T^{k}(\widehat{\delta}-\widehat{\tau}) \in[-n, n] \times[-m, m] .
$$

Consequently,

$$
q T^{k}(\widehat{\delta}-\widehat{\tau}) \in[-q n, q n] \times[-q m, q m] .
$$

Then, for any $\tau \in M_{k}, R_{k}(\tau) \subset(([-q n, q n] \times[-q m, q m]) \cap$ $\left.\mathbb{Z}^{2}\right) / q$. So the set $R$ is finite.

Proof of Theorem 1. It is a direct conclusion from Theorem 6 and Proposition 7. 


\section{Examples}

Example 1. Let $T=\left(\begin{array}{ll}4 & 0 \\ 0 & 3\end{array}\right)$ and $D=\left\{d_{1}, d_{2}, d_{3}, d_{4}\right\}$, where $d_{1}=$ $\left(\begin{array}{l}0 \\ 0\end{array}\right), d_{2}=\left(\begin{array}{l}3 \\ 0\end{array}\right), d_{3}=\left(\begin{array}{l}1 \\ 1\end{array}\right)$, and $d_{4}=\left(\begin{array}{l}3 \\ 2\end{array}\right) . t=(1 / 3,1 / 4)$.

By Proposition 7, the set $R$ is finite. The types of the basic rectangles can be determined by (15). It is clear that $R_{0}(\Omega)=t$, denoted by $A$. For $k=1$, among four offsprings of $\Omega$, only $\Omega_{3}$ and $\Omega_{4}$ have nonempty neighborhood, which are connected by edges $(1,1)$ and $(3,2)$ from their parent $\Omega$, respectively. By (15), they are of different types; denote them symbolically as

$$
A \stackrel{(1,1)}{\longrightarrow} B, \quad A \stackrel{(3,2)}{\longrightarrow} C .
$$

For $k=2$, direct computation yields

$$
\Omega_{32}, \Omega_{41}, \Omega_{43} \in \mathscr{M}_{2} .
$$

They are of new types and $\Omega_{32}, \Omega_{43}$ are of the same type. So we have

$$
B \stackrel{(3,0)}{\longrightarrow} D, \quad C \stackrel{(1,1)}{\longrightarrow} D, \quad C \stackrel{(0,0)}{\longrightarrow} E .
$$

Above computation process can also be seen from Figure 1. Upon the third iteration, none of the offsprings of $\Omega_{41}$ belongs to $\mathscr{M}_{3}$; then type $E$ and all paths going to $E$ are discarded.

By the same argument as above, we have

$$
D \stackrel{(0,0)}{\longrightarrow} F, \quad D \stackrel{(1,1)}{\longrightarrow} C .
$$

However none of the offsprings of $\Omega_{321}$, whose type is of $F$, belongs to $\mathscr{M}_{4}$. Then type $F$ and all paths going to $F$ are deleted. By Proposition 3, the above process exhausts all possible types and yields the adjacency matrix

$$
S=\left(\begin{array}{llll}
0 & 1 & 1 & 0 \\
0 & 0 & 0 & 1 \\
0 & 0 & 0 & 1 \\
0 & 0 & 1 & 0
\end{array}\right),
$$

and by Kenyon-Peres's Theorem, we have

$$
\begin{aligned}
& S_{0}=\left(\begin{array}{llll}
0 & 0 & 0 & 0 \\
0 & 0 & 0 & 1 \\
0 & 0 & 0 & 0 \\
0 & 0 & 0 & 0
\end{array}\right), \\
& S_{1}=\left(\begin{array}{llll}
0 & 1 & 0 & 0 \\
0 & 0 & 0 & 0 \\
0 & 0 & 0 & 1 \\
0 & 0 & 1 & 0
\end{array}\right), \\
& S_{2}=\left(\begin{array}{llll}
0 & 0 & 1 & 0 \\
0 & 0 & 0 & 0 \\
0 & 0 & 0 & 0 \\
0 & 0 & 0 & 0
\end{array}\right) .
\end{aligned}
$$

Example 2. Let $T=\left(\begin{array}{ll}5 & 0 \\ 0 & 4\end{array}\right)$ and $D=\left\{d_{1}, d_{2}, d_{3}, d_{4}\right\}$, where $d_{1}=\left(\begin{array}{l}0 \\ 0\end{array}\right), d_{2}=\left(\begin{array}{l}4 \\ 0\end{array}\right), d_{3}=\left(\begin{array}{l}2 \\ 1\end{array}\right)$, and $d_{4}=\left(\begin{array}{l}3 \\ 2\end{array}\right) . t=$ $(12 / 25,5 / 16)$.

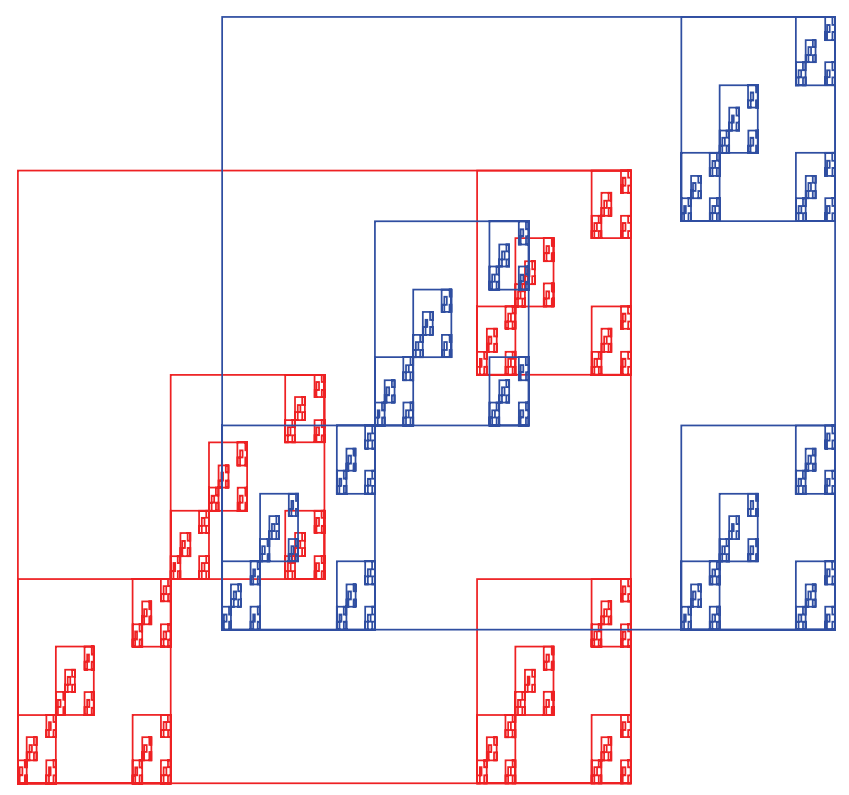

Figure 1: Construction of $K \cap K+t$. Red and blue rectangles denote the construction of $K$ and $K+t$, respectively.

Again by Proposition 7, the set $R$ is finite. First, $R_{0}(\Omega)=t$; denote it by $A$. For $k=1, \Omega_{3}, \Omega_{4} \in \mathscr{M}_{1}$, and $N\left(\Omega_{3}\right)=\left\{\Omega_{1}^{t}\right\}$, $N\left(\Omega_{4}\right)=\left\{\Omega_{1}^{t}\right\}$. They are of different types and then denoted by

$$
A \stackrel{(2,1)}{\longrightarrow} B, \quad A \stackrel{(3,2)}{\longrightarrow} C .
$$

For $k=2, \Omega_{33}, \Omega_{34}, \Omega_{41} \in \mathscr{M}_{2}$, and $N\left(\Omega_{33}\right)=\left\{\Omega_{11}^{t}\right\}$, $N\left(\Omega_{34}\right)=\left\{\Omega_{11}^{t}, \Omega_{13}^{t}\right\}, N\left(\Omega_{41}\right)=\left\{\Omega_{14}^{t}\right\}$. Their types are of $D$, $E$, and $F$, respectively. Thus we have

$$
B \stackrel{(2,1)}{\longrightarrow} D, \quad B \stackrel{(3,2)}{\longrightarrow} E, \quad C \stackrel{(0,0)}{\longrightarrow} F .
$$

Upon the third iteration, $\Omega_{33}$ produces four offsprings in $\mathscr{M}_{3}$, which yields

$$
D \stackrel{(0,0)}{\longrightarrow} D, \quad D \stackrel{(2,1)}{\longrightarrow} G, \quad D \stackrel{(3,2)}{\longrightarrow} H, \quad D \stackrel{(4,0)}{\longrightarrow} D .
$$

Only one offspring of $\Omega_{34}$, whose type is of $E$, belongs to $\mathscr{M}_{3}$; then we get

$$
E \stackrel{(4,0)}{\longrightarrow} I .
$$

None of the offsprings of $\Omega_{41}$, whose type is $F$, belongs to $\mathscr{M}_{3}$; then type $F$ and all paths going to $F$ are discarded. For $k=4$, by the same argument as above, we have

$$
\begin{array}{rlll}
G \stackrel{(0,0)}{\longrightarrow} D, & G \stackrel{(2,1)}{\longrightarrow} G, & G \stackrel{(3,2)}{\longrightarrow} H, & G \stackrel{(4,0)}{\longrightarrow} D, \\
H \stackrel{(0,0)}{\longrightarrow} D, & H \stackrel{(2,1)}{\longrightarrow} D, & H \stackrel{(3,2)}{\longrightarrow} H, & H \stackrel{(4,0)}{\longrightarrow} D,
\end{array}
$$

$$
I \stackrel{(4,0)}{\longrightarrow} I .
$$




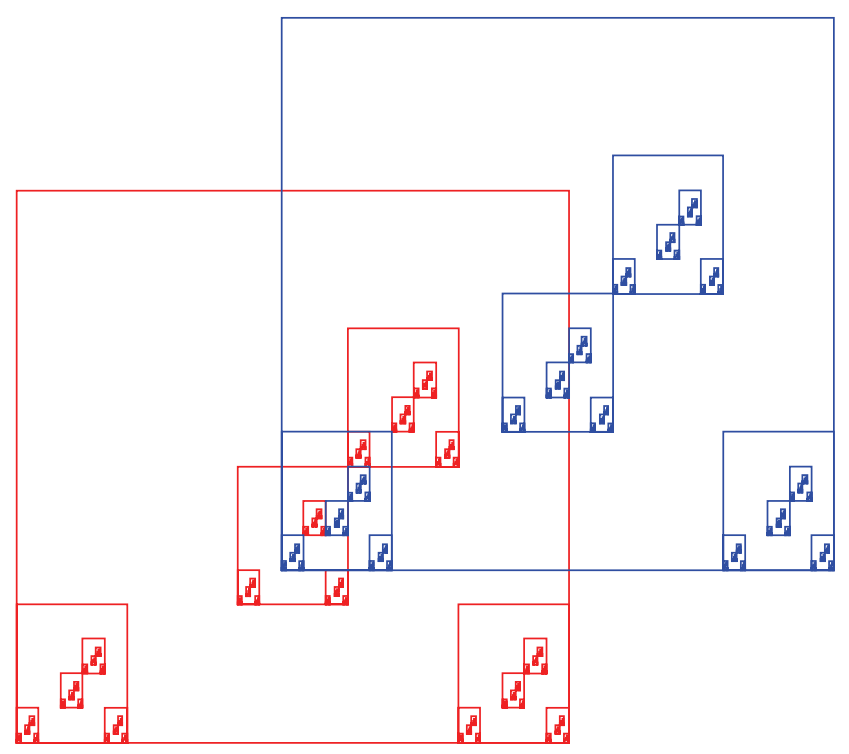

Figure 2: Construction of $K \cap K+t$. Red and blue rectangles denote the construction of $K$ and $K+t$, respectively.

Figure 2 can avail to understand the above computation process. By Proposition 3, these are all the types of the intersection and the adjacency matrix is

$$
S=\left(\begin{array}{lllllll}
0 & 1 & 0 & 0 & 0 & 0 & 0 \\
0 & 0 & 1 & 1 & 0 & 0 & 0 \\
0 & 0 & 2 & 0 & 1 & 1 & 0 \\
0 & 0 & 0 & 0 & 0 & 0 & 1 \\
0 & 0 & 2 & 0 & 1 & 1 & 0 \\
0 & 0 & 2 & 0 & 1 & 1 & 0 \\
0 & 0 & 0 & 0 & 0 & 0 & 1
\end{array}\right)
$$

Also by Kenyon-Peres's Theorem, we get

$$
\begin{aligned}
S_{0} & =\left(\begin{array}{lllllll}
0 & 0 & 0 & 0 & 0 & 0 & 0 \\
0 & 0 & 0 & 0 & 0 & 0 & 0 \\
0 & 0 & 2 & 0 & 0 & 0 & 0 \\
0 & 0 & 0 & 0 & 0 & 0 & 1 \\
0 & 0 & 2 & 0 & 0 & 0 & 0 \\
0 & 0 & 2 & 0 & 0 & 0 & 0 \\
0 & 0 & 0 & 0 & 0 & 0 & 1
\end{array}\right), \\
S_{1} & =\left(\begin{array}{lllllll}
0 & 1 & 0 & 0 & 0 & 0 & 0 \\
0 & 0 & 1 & 0 & 0 & 0 & 0 \\
0 & 0 & 0 & 0 & 1 & 0 & 0 \\
0 & 0 & 0 & 0 & 0 & 0 & 0 \\
0 & 0 & 0 & 0 & 1 & 0 & 0 \\
0 & 0 & 0 & 0 & 1 & 0 & 0 \\
0 & 0 & 0 & 0 & 0 & 0 & 0
\end{array}\right),
\end{aligned}
$$

$$
\begin{aligned}
& S_{2}=\left(\begin{array}{lllllll}
0 & 0 & 0 & 0 & 0 & 0 & 0 \\
0 & 0 & 0 & 1 & 0 & 0 & 0 \\
0 & 0 & 0 & 0 & 0 & 1 & 0 \\
0 & 0 & 0 & 0 & 0 & 0 & 0 \\
0 & 0 & 0 & 0 & 0 & 1 & 0 \\
0 & 0 & 0 & 0 & 0 & 1 & 0 \\
0 & 0 & 0 & 0 & 0 & 0 & 0
\end{array}\right), \\
& S_{3}=\left(\begin{array}{lllllll}
0 & 0 & 0 & 0 & 0 & 0 & 0 \\
0 & 0 & 0 & 0 & 0 & 0 & 0 \\
0 & 0 & 0 & 0 & 0 & 0 & 0 \\
0 & 0 & 0 & 0 & 0 & 0 & 0 \\
0 & 0 & 0 & 0 & 0 & 0 & 0 \\
0 & 0 & 0 & 0 & 0 & 0 & 0 \\
0 & 0 & 0 & 0 & 0 & 0 & 0
\end{array}\right) .
\end{aligned}
$$

\section{Acknowledgments}

The authors would like to thank the anonymous referees for their helpful comments and suggestions that led to a significant improvement of the paper. This work was supported by the National Natural Science Foundation of China (nos. 61003178, 11201312, 61070087, 61373087, 11071150, and 61272252) and by the Municipal Science and Technology Plan of Shenzhen in China (JC201105170615A, JC201005280508A).

\section{References}

[1] C. McMullen, "The Hausdorff dimension of general Sierpiński carpets," Nagoya Mathematical Journal, vol. 96, pp. 1-9, 1984.

[2] T. Bedford, Crinkly curves, Markov partitions and box dimension in self-similar sets [Ph.D. thesis], University of Warwick, 1984.

[3] Y. Peres, "The packing measure of self-affine carpets," Mathematical Proceedings of the Cambridge Philosophical Society, vol. 115, no. 3, pp. 437-450, 1994.

[4] Y. Peres, "The self-affine carpets of McMullen and Bedford have infinite Hausdorff measure," Mathematical Proceedings of the Cambridge Philosophical Society, vol. 116, no. 3, pp. 513-526, 1994.

[5] R. Kenyon and Y. Peres, "Measures of full dimension on affineinvariant sets," Ergodic Theory and Dynamical Systems, vol. 16, no. 2, pp. 307-323, 1996.

[6] R. Kenyon and Y. Peres, "Hausdorff dimensions of sofic affineinvariant sets," Israel Journal of Mathematics, vol. 94, pp. 157$178,1996$.

[7] Y. Gui and W. Li, "Hausdorff dimension of fiber-coding subSierpinski carpets," Journal of Mathematical Analysis and Applications, vol. 331, no. 1, pp. 62-68, 2007.

[8] S. Hua, "Dimensions of self-affine sets with overlaps," Chinese Annals of Mathematics B, vol. 24, no. 3, pp. 275-284, 2003.

[9] R. Kenyon and Y. Peres, "Intersecting random translates of invariant Cantor sets," Inventiones Mathematicae, vol. 104, no. 3, pp. 601-629, 1991.

[10] R. F. Williams, "How big is the intersection of two thick Cantor sets?, in Contemporary Mathematics," in Proceedings of the Joint Summer Research Conference on Continua and Dynamical Systems, M. Brown, Ed., vol. 117, pp. 163-175, A.M.S., Providence, RI, USA, 1991. 
[11] R. L. Kraft, "Intersection of thick Cantor sets," Memoirs: American Mathematical Society, vol. 97, no. 468, 1992.

[12] G. J. Davis and T. Y. Hu, "On the structure of the intersection of two middle third Cantor sets," Publicacions Matemàtiques, vol. 39, no. 1, pp. 43-60, 1995.

[13] W. Li and D. Xiao, "On the intersection of translation of middle$\alpha$ Cantor sets," in Fractals and Beyond-Complexities in the Sciences, pp. 137-148, World Scientific, Singapore, 1998.

[14] F. Nekka and J. Li, "Intersection of triadic Cantor sets with their translates. I. Fundamental properties," Chaos, Solitons and Fractals, vol. 13, no. 9, pp. 1807-1817, 2002.

[15] J. Li and F. Nekka, "Intersection of triadic Cantor sets with their translates. II. Hausdorff measure spectrum function and its introduction for the classification of Cantor sets," Chaos, Solitons and Fractals, vol. 19, no. 1, pp. 35-46, 2004.

[16] M. Dai and L. Tian, "Intersection of the Sierpinski carpet with its rational translate," Chaos, Solitons \& Fractals, vol. 31, no. 1, pp. 179-187, 2007.

[17] M. Dai and L. Tian, "Some properties for the intersection of Moran sets with their translates," Chaos, Solitons \& Fractals, vol. 31, no. 3, pp. 757-764, 2007.

[18] Y. Zou, W. Li, and C. Yan, "Intersecting nonhomogeneous Cantor sets with their translations," Nonlinear Analysis. Theory, Methods \& Applications, vol. 74, no. 14, pp. 4660-4670, 2011.

[19] R. L. Kraft, "Random intersections of thick Cantor sets," Transactions of the American Mathematical Society, vol. 352, no. 3, pp. 1315-1328, 2000.

[20] S. Hua and H. Rao, "Graph-directed structures of self-similar sets with overlaps," Chinese Annals of Mathematics B, vol. 21, no. 4, pp. 403-412, 2000.

[21] X.-G. He, K.-S. Lau, and H. Rao, "Self-affine sets and graphdirected systems," Constructive Approximation, vol. 19, no. 3, pp. 373-397, 2003.

[22] S.-M. Ngai and Y. Wang, "Hausdorff dimension of self-similar sets with overlaps," Journal of the London Mathematical Society, vol. 63, no. 3, pp. 655-672, 2001. 


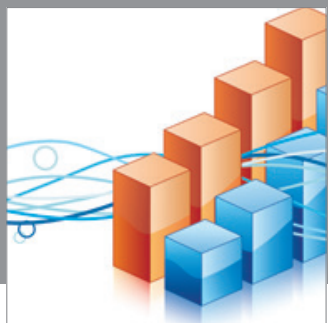

Advances in

Operations Research

mansans

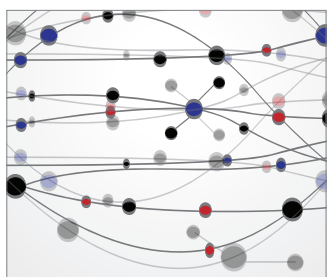

The Scientific World Journal
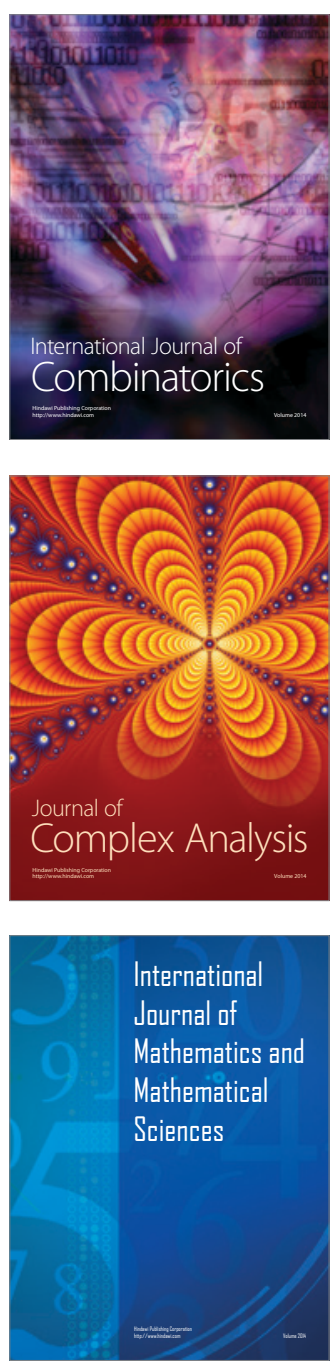
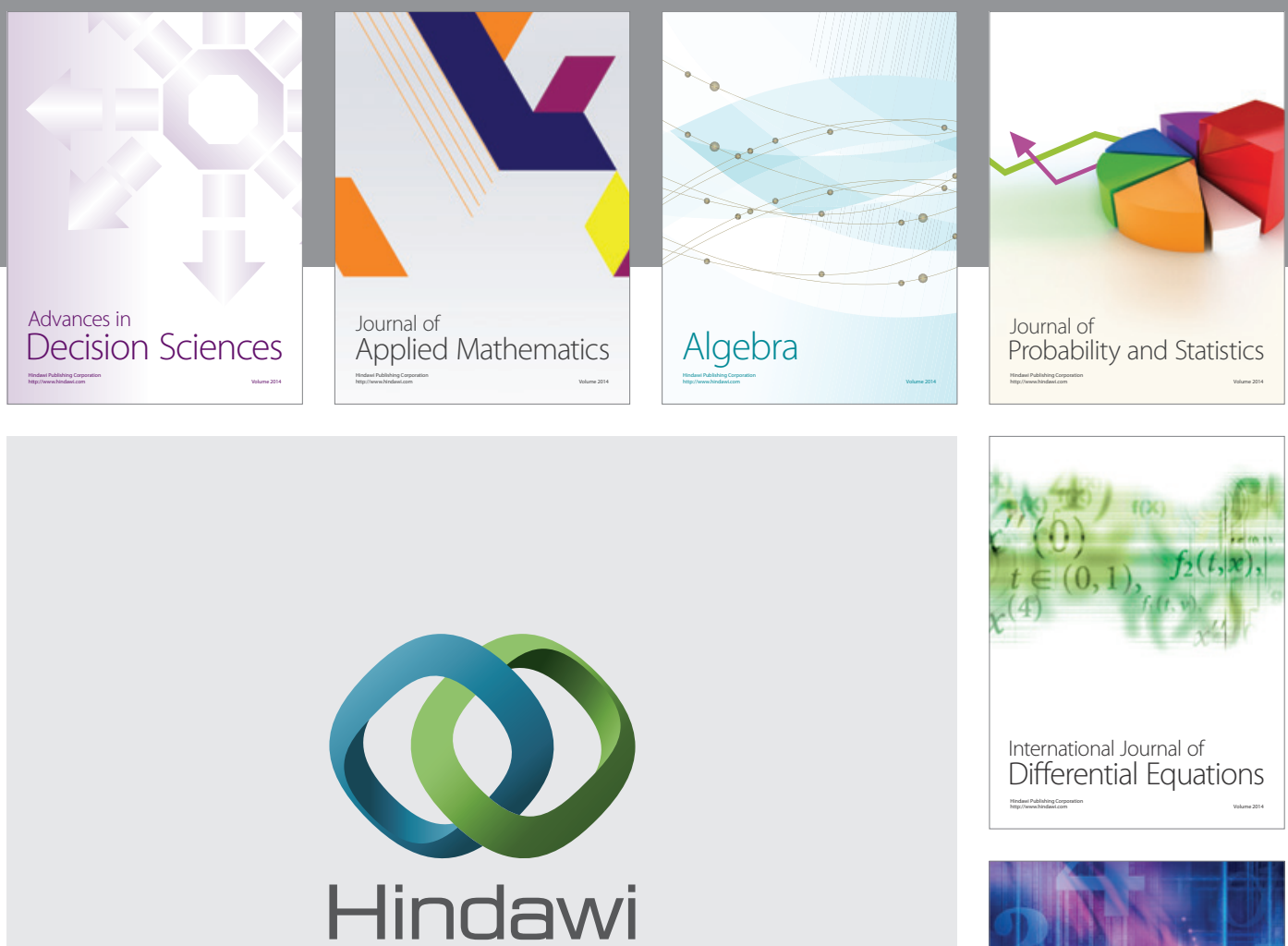

Submit your manuscripts at http://www.hindawi.com
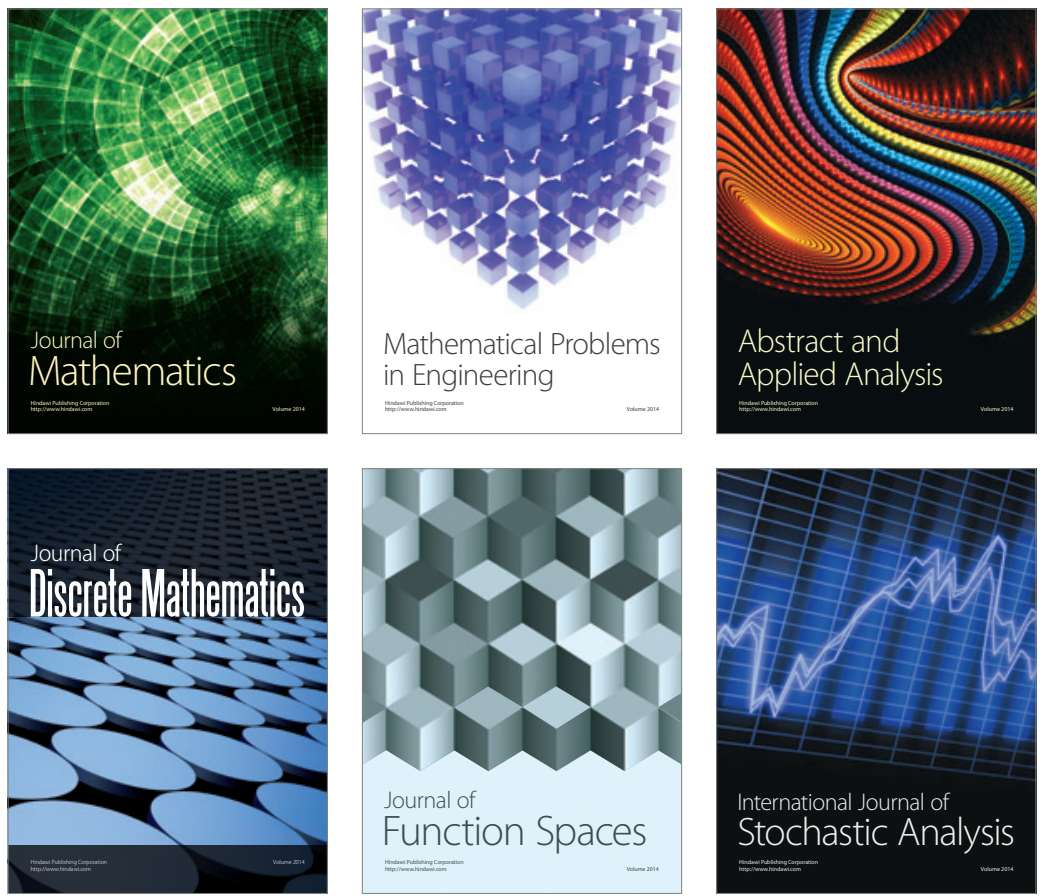

Journal of

Function Spaces

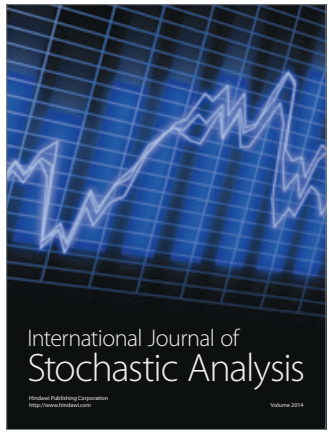

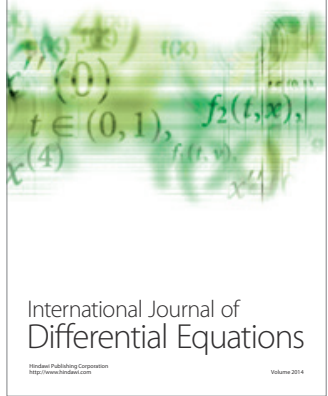
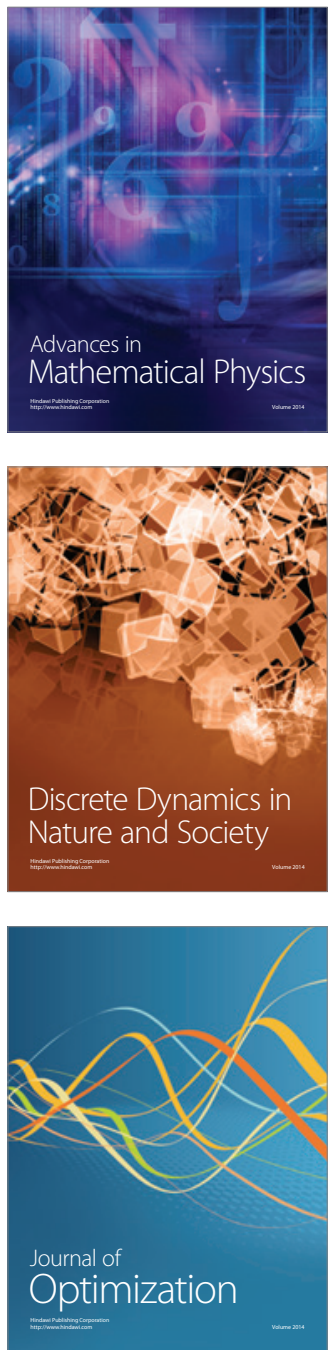